\title{
Quasiperiodicity and Mode Locking of Undriven Spontaneous Oscillations in Germanium Crystals
}

\author{
J. Peinke, J. Parisi, B. Röhricht, B. Wessely, and K. M. Mayer \\ Physikalisches Institut II, Universität Tübingen, Tübingen, Fed. Rep. Germany
}

Z. Naturforsch. 42 a, $841-845$ (1987); received May 7, 1987

\begin{abstract}
Nonlinear current transport behavior during low-temperature avalanche breakdown of germanium comprises the spontaneous formation of spatial and temporal dissipative structures in the formerly homogeneous semiconductor. Due to the simultaneous presence of two and more competing fundamental oscillatory modes intrinsic to our synergetic semiconductor system, selforganized emergence of quasiperiodic and mode-locked states can be observed followed by the quasiperiodic approach to low-dimensional chaos.
\end{abstract}

Intensively growing interest in computer studies in nonlinear science together with the recent discovery of universal criterions for similar nonlinear dynamical processes have inspired experimentalists to characterize their systems in a new way. In particular, numerical calculations based on the simple circle-map formalism [1] have resulted in quantitative predictions for certain universal properties of the quasiperiodic approach to turbulence. Nonlinear dissipative dynamical systems in the presence of two or more competing fundamental oscillatory modes with originally incommensurate frequencies are expected to exhibit quasiperiodicity and modelocking phenomena prior to the onset of low-dimensional chaos. Increasing strength of nonlinear coupling between the oscillators develops an increasing tendency to lock into commensurate motion where the ratio of the oscillatory frequencies is a rational number. Transition to chaos can be caused by interaction and overlap of mode-locked resonances [1]. So far, a number of these predicted features have been tested in different experimental systems [2-4]. Best conformity with numerical results of the circle map has been obtained in experiments where an external periodic forcing is applied to a single internal oscillatory mode of the system. As an advantage, the amplitude and frequency of the driving force represent adequate control parameters which are simply related to the parameters of the

Reprint requests to J. Parisi, Physikalisches Institut II, Universität Tübingen, Morgenstelle 14, D-7400 Tübingen, F.R.G. circle map. However, an external periodic forcing does not allow certain types of quasiperiodic transitions [2].

This paper reports on experimental investigations of the self-organized emergence of quasiperiodic and mode-locked behavior during impact-ionization-induced avalanche breakdown of germanium at low temperatures. One distinct feature of our work is the presence of two and more natural oscillatory modes intrinsic to our semiconductor system as a consequence of the spatio-temporal nonlinear current transport in the breakdown regime. Our experiments deal with a synergetic system, consisting of spatially separated oscillation centers arising spontaneously and interacting in a nonlinear way. Depending sensitively upon such control parameters as the applied d.c. electric and magnetic field, these undriven spontaneous current oscillations are demonstrated to undergo a phase transition from a stable fixed point via periodic and quasiperiodic states of two incommensurate frequencies to lowdimensional chaos. The two-frequency quasiperiodic state is found to be interrupted by distinct modelocking intervals.

Our experiments were performed at $2.10 \mathrm{~K}$ on homogeneously doped p-type germanium single crystals (acceptor concentration of about $10^{14} \mathrm{~cm}^{-3}$ ) having the dimensions of about $(0.25 \times 2.0 \times 4.4) \mathrm{mm}^{3}$ and carrying properly arranged ohmic aluminum contacts. The sample geometry and the electronic measuring configuration is shown schematically in Figure 1. An electric field was applied to the outer contacts (d.c. bias voltage $V_{0}$ ). A d.c. magnetic field 


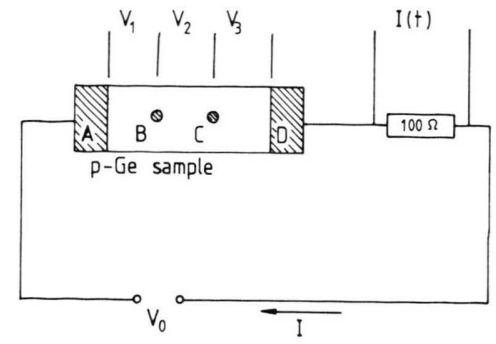

Fig. 1. Scheme of the experimental set-up. The hatched areas $\mathrm{A}-\mathrm{D}$ upon the $\mathrm{Ge}$ sample indicate the evaporated ohmic $\mathrm{Al}$ contacts.

perpendicular to the broad sample surfaces could also be applied using a superconducting coil surrounding a copper metal shield employed for protection against external irradiation. The inner probe contacts (of about $0.2 \mathrm{~mm}$ diameter) served for monitoring independently the partial voltages $V_{1}$, $V_{2}$, and $V_{3}$ along the sample. The current $I$ was found from the voltage drop upon the $100 \Omega$ load resistor. Further details on the experimental techniques can be found elsewhere [5].

Similar to the corresponding phenomena in gaseous plasma discharges, impact ionization of the shallow impurities can be achieved in the bulk of the homogeneously doped semiconductor at low temperatures. In the temperature regime of liquid helium most of the charge carriers are frozen out at the impurities. Since the ionization energy is only about $10 \mathrm{meV}$ and electron-phonon scattering is strongly reduced, avalanche breakdown already takes place at electric fields of a few $\mathrm{V} / \mathrm{cm}$ and persists until all impurities are ionized. The underlying nonequilibrium phase transition from a low conducting state to a high conducting state is directly reflected in strongly nonlinear regions of negative differential resistivity. Under slight variation of distinct control parameters (electric field, magnetic field, temperature) the resulting electric current flow displays a wide variety of spatio-temporal nonlinear transport behavior, comprising the spontaneous formation of both spatial and temporal dissipative structures in the formerly homogeneous semiconductor [5-7].

In the following, we concentrate on the quasiperiodic and mode-locked behavior of undriven, self-generated current oscillations (with a relative amplitude of about $10^{-3}$ in the frequency range

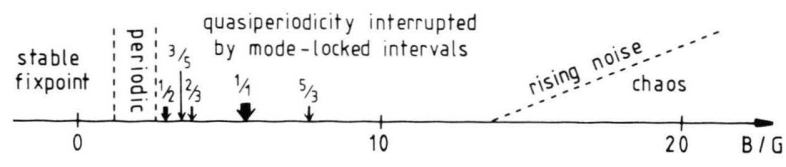

Fig. 2. Scheme of the examined quasiperiodic transition to chaos via intervening mode-locking states. The major stable resonances locked at the frequency ratios $1: 2,3: 5$, $2: 3,1: 1$, and $5: 3$ over finite regions of the applied magnetic control field $(40 \mathrm{mG}, 5 \mathrm{mG}, 10 \mathrm{mG}, 80 \mathrm{mG}$, and $10 \mathrm{mG}$, respectively) are indicated by arrows.

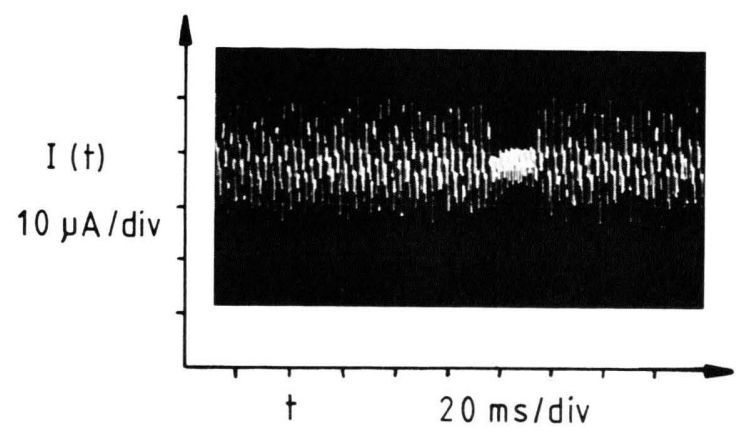

Fig. 3. Time profile of the spontaneous current oscillations at the onset of quasiperiodicity $(B=2.56 \mathrm{G})$.

$0.1-10 \mathrm{kHz}$ ), superimposed upon the steady d.c. current (of typically a few $\mathrm{mA}$ ) in the strongly nonlinear post-breakdown regime of the currentvoltage characteristic. Figure 2 gives a schematic synopsis of the examined transition from an initial nonoscillating state (fixed point) to a final noisy (chaotic) state as a function of the applied transverse magnetic field. The bias voltage was always kept at the constant value $V_{0}=2.3280 \mathrm{~V}$. Increasing the magnetic control field from $B=0 \mathrm{G}$ up to $B=1.24 \mathrm{G}$, the steady-state flow of the system trajectory bifurcates from the stable fixed point to spontaneous limit-cycle oscillations. At $B=2.56 \mathrm{G}$ the oscillatory system flow undergoes an intermittentlike transition of stochastically switching between periodicity with a single intrinsic frequency and quasiperiodicity with two incommensurate intrinsic frequencies. The corresponding temporal structure of the spontaneous current oscillations is shown in Figure 3.

Since the system trajectory of the two-frequency quasiperiodic state traces out an invariant two-torus 


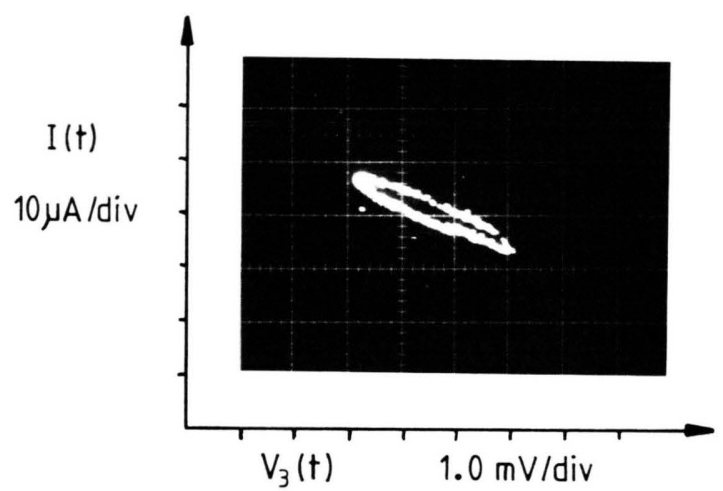

Fig. 4. Poincare section of the quasiperiodic state $(B=$ $8.57 \mathrm{G})$. The construction is described in the text.

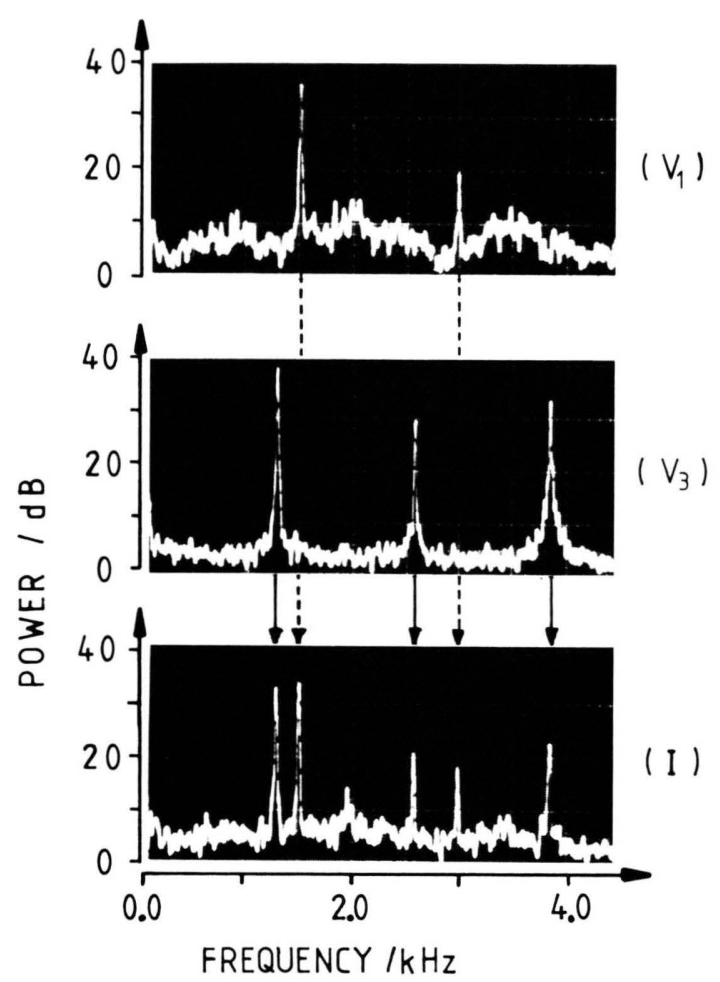

Fig. 5. Power spectra of the partial voltages $V_{1}$ and $V_{3}$ and the total current $I$ at the quasiperiodic state $(B=7.22 \mathrm{G}$, $1 \mathrm{k} \Omega$ load resistor).

in phase space, the underlying quasiperiodic attractor can be characterized by constructing Poincare sections on the basis of real-time phase plots in the following way. First, one projected 2-D phase portrait is obtained from the measured current signal $I(t)$ plotted versus a distinct partial voltage drop
$V_{i}(t)$ (say, $i=3$ ) on the $x-y$ screen of an oscilloscope. The brightness ( $z$-axis) of the phase portrait is then modulated by means of appropriate voltage pulses, obtained from a pulse generator which is triggered by a second partial voltage signal $V_{i}(t)$ (say, $j=1$ ). As displayed in Fig. 4, the typically resulting even distribution of the bright cross-section points along an unfolded, closed loop clearly indicates quasiperiodic motion of the phase-space trajectory on the surface of the toroidal attractor. Additional information on the weak nonlinear coupling of the corresponding two fundamental oscillatory modes could be obtained by simultaneously recording the power spectra of different partial voltage drops $V_{i}$ and of the total sample current I. As a result, Fig. 5 obviously demonstrates independent temporal behavior of the two voltages $V_{1}$ and $V_{3}$, confirming the model that the above twofrequency quasiperiodic current flow originates from a superposition of two spatially separated oscillation centers.

Under variation of the external magnetic field, the two fundamental intrinsic frequencies of the quasiperiodic state are changing. Whenever the frequency of a harmonic of one oscillator approaches some harmonic of the other, the originally incommensurate frequencies of the two oscillators lock exactly into rational ratios according to the wellknown hierarchical Farey-tree ordering [1]. Depending upon the degree of nonlinear coupling, the resulting temporally resonant mode-locking phenomenon extends over some finite regions of control parameter space. For illustration, Fig. 6 shows the phase portrait of one of the major stable locked states observed in our system (cf. Fig. 2) together with the phase portrait of the quasiperiodic state. The intersection points of the corresponding Poincare map are the bright spots at the lower edge of the phase plots. As a further example, Fig. 7 displays the magnetic field dependence of the two individual frequencies approaching the 1:1 modelocking interval which is most elongated. The curvature at the edges of the locked region clearly illustrates the self-organized, strong mutual interaction between the two competing oscillatory modes (in contrast to the case of an externally driven single-mode oscillator [4]).

Finally, we emphasize a further structural change of the temporal system behavior at increased magnetic control field. Compared to the line-like char- 

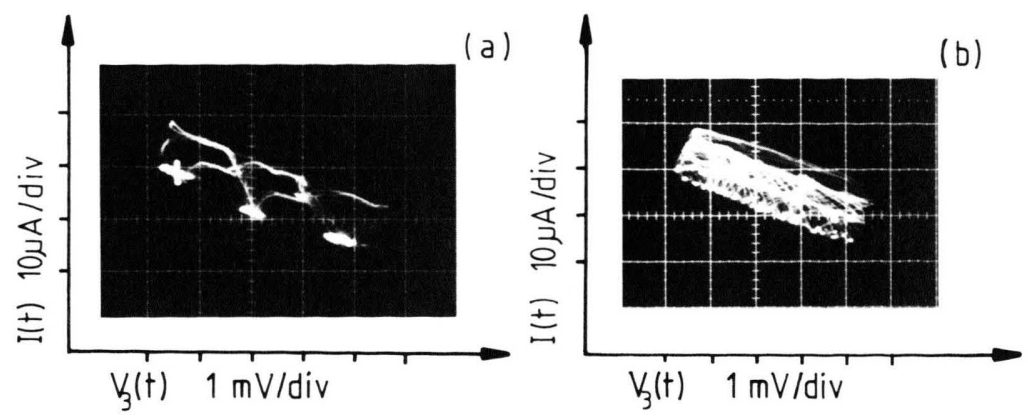

Fig. 6. Phase portraits of (a) the $2: 3$ locked state $(B=3.74 \mathrm{G})$ and $(\mathrm{b})$ the quasiperiodic state $(B=4.13 \mathrm{G})$.

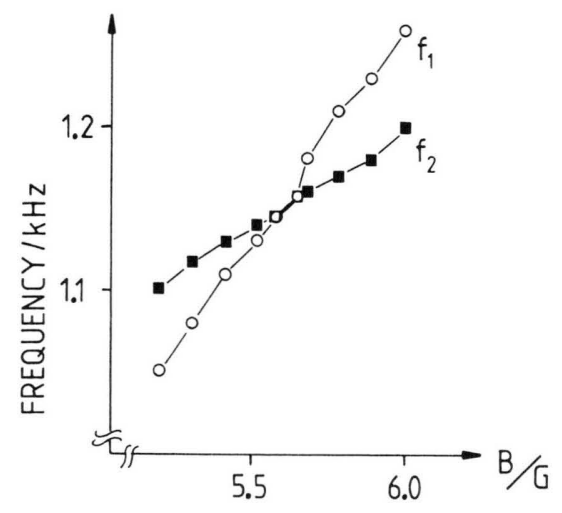

Fig. 7. Dependence of the two fundamental oscillatory modes (frequencies $f_{1}, f_{2}$ ) upon the applied magnetic control field in the vicinity of the $1: 1$ locked state.

acter of the Poincare map of the two-frequency quasiperiodic state shown in Fig. 4, the cross-section of the trajectorial flow in Fig. 8 a becomes no longer smooth. The toroidal attractor now occupies an extended volume in phase space, signalling the break-up of the invariant two-torus. The corresponding increase of the degree of nonlinear coupling is further manifested in the growing strength and number of high-order mixing frequencies found at magnetic fields beyond $B=13.5 \mathrm{G}$. Accordingly, the broadband noise level gradually rises, and somewhat broadened spectral peaks remain in the current power spectrum (see Fig. $8 \mathrm{~b}$ as an example). Following the argumentation introduced by Ruelle, Takens, and Newhouse [8], the above transition can be identified as the quasiperiodic approach to lowdimensional chaos.

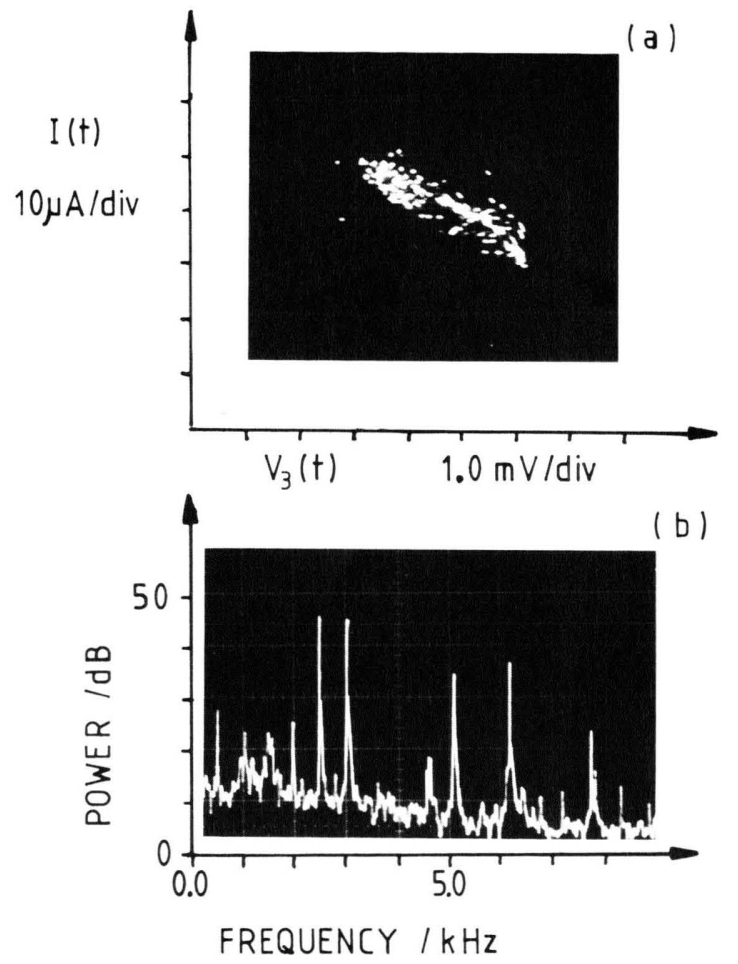

Fig. 8. (a) Poincare section and (b) spectral noise power of the current $I$ at the onset of chaos $(B=19.95 \mathrm{G})$.

\section{Acknowledgements}

We are pleased to thank R. P. Huebener for critically reading the paper, and O. E. Rössler, C. Jeffries, R. M. Westervelt, S. W. Teitsworth, P. Bryant, E Schöll, W. Prettl, and U. Rau for helpful discussions. This work was supported by a grant from the Stiftung Volkswagenwerk. 
[1] M. H. Jensen, P. Bak, and T. Bohr, Phys. Rev. A 30, 1960 (1984); - T. Bohr, P. Bak, and M. H. Jensen, Phys. Rev. A 30, 1970 (1984); - P. Bak, T. Bohr, and M. H. Jensen, Phys. Scr. T 9, 50 (1985); - P. Cvitanovic, M. H. Jensen, L. P. Kadanoff, and I. Procaccia, Phys. Rev. Lett. 55, 343 (1985).

[2] For experiments on hydrodynamic systems see: R. W. Wolden, P. Kolodner, A. Passner, and C. M. Surko, Phys. Rev. Lett. 53, 242 (1984); - J. Stavans, F. Heslot, and A. Libchaber, Phys. Rev. Lett. 55, 596 (1985); - A. P. Fein, M. S. Heutmaker, and J. P. Gollub, Phys. Scr. T 9, 79 (1985); - H. Haucke and R. Ecke, Physica D (to be published).

[3] For experiments on chemical systems see: H. L. Swinney and J. Maselko, Phys. Rev. Lett. 55, 2366 (1985).
[4] For experiments on solid-state systems see: G. A Held and C. Jeffries, Phys. Rev. Lett. 56, 1183 (1986); S. Martin and W. Martienssen, Phys. Rev. Lett. 56, 1522 (1986); - E. G. Gwinn and R. M. Westervelt, Phys. Rev. Lett. 57, 1060 (1986).

[5] J. Peinke, A. Mühlbach, R. P. Huebener, and J. Parisi, Phys. Lett. 108 A, 407 (1985).

[6] J. Peinke, B. Röhricht, A. Mühlbach, J. Parisi, Ch. Nöldeke, R. P. Huebener, and O. E. Rössler, Z. Naturforsch. 40 a, 562 (1985).

[7] K. M. Mayer, R. Gross, J. Parisi, J. Peinke, and R. P. Huebener, Solid State Commun. 63, 55 (1987).

[8] D. Ruelle and F. Takens, Commun. Math. Phys. 20, 167 (1971); - S. Newhouse, D. Ruelle, and F. Takens, Commun. Math. Phys. 64, 35 (1978). 